OPEN ACCESS

Edited by:

Shiv K. Gupta,

Mayo Clinic, United States

Reviewed by:

Karishma Rajani,

Mayo Clinic, United States

Sujuan Guo,

Dana-Farber Cancer Institute,

United States

${ }^{*}$ Correspondence:

Massimo Romani

tumor.epigenetics@gmail.com;

massimo.romani@hsanmartino.it

Specialty section:

This article was submitted to

Cancer Molecular Targets and

Therapeutics,

a section of the journal

Frontiers in Oncology

Received: 26 July 2018 Accepted: 02 October 2018

Published: 23 October 2018

Citation:

Romani M, Pistillo MP, Carosio R,

Morabito A and Banelli B (2018) Immune Checkpoints and Innovative

Therapies in Glioblastoma.

Front. Oncol. 8:464.

doi: 10.3389/fonc.2018.00464

\section{Immune Checkpoints and Innovative Therapies in Glioblastoma}

\author{
Massimo Romani ${ }^{1 *}$, Maria Pia Pistillo ${ }^{1}$, Roberta Carosio ${ }^{1}$, Anna Morabito $^{1}$ and \\ Barbara Banelli ${ }^{1,2}$
}

${ }^{1}$ Laboratory of Tumor Epigenetics, IRCCS Ospedale Policlinico San Martino, Genova, Italy, ${ }^{2}$ Department of Health Sciences, University of Genova, Genova, Italy

Targeting the Immune Checkpoint molecules (ICs; CTLA-4, PD-1, PD-L1/2, and others) which provide inhibitory signals to T cells, dramatically improves survival in hard-to-treat tumors. The establishment of an immunosuppressive environment prevents endogenous immune response in glioblastoma; therefore, manipulating the host immune system seems a reasonable strategy also for this tumor. In glioma patients the accumulation of $\mathrm{CD}^{+} / \mathrm{CD}^{+}+\mathrm{T}$ cells and Treg expressing high levels of CTLA-4 and PD-1, or the high expression of PD-L1 in glioma cells correlates with WHO high grade and short survival. Few clinical studies with IC inhibitors (ICis) were completed so far. Notably, the first large-scale randomized trial (NCT 02017717) that compared PD-1 blockade and anti-VEGF, did not show an OS increase in the patients treated with anti-PD-1. Several factors could have contributed to the failure of this trial and must be considered to design further clinical studies. In particular the possibility of targeting at the same time different ICs was pre-clinically tested in an animal model were inhibitors against IDO, CTLA-4 and PD-L1 were combined and showed persistent and significant antitumor effects in glioma-bearing mice. It is reasonable to hypothesize that the immunological characterization of the tumor in terms of type and level of expressed IC molecules on the tumor and TIL may be useful to design the optimal ICi combination for a given subset of tumor to overcome the immunosuppressive milieu of glioblastoma and to efficiently target a tumor with such high cellular complexity.

Keywords: glioblastoma, therapy, immune checkpoint, CTLA-4, PD-1, PD-L1

\section{INTRODUCTION}

Since the discovery in 2005 of the clinical utility of Temozolomide in glioblastoma (GBM) patients (1), no other cytotoxic drug was added in the standard treatment protocols. In the meantime our knowledge on the molecular mechanisms deranged in GBM has had impressive advancements and the possibility of targeting these pathways has been extensively exploited in the hope to improve the current standard of care (2-4). Differently from many other tumors, in GBM the promises of molecularly targeted therapies against oncogenic alterations did not meet success in phase I/II and III trials even though they were highly promising in preclinical models; thereafter they have limited clinical utilization (5). The lack of success of targeted therapies and the limited activity of standard cytotoxic treatments in GBM, reside in the cellular complexity and clonal evolution of this tumor $(6,7)$. Moreover, many molecules that display strong antitumor activity in vitro against glioma cells and that are utilized for the therapy of other tumors, are ineffective in vivo because they cannot pass through the Blood Brain Barrier (BBB), or because of drug efflux, intrinsic or 
rapidly developing drug resistance and, last but not least, the presence of a pool of cancer cells with stemness characteristics (7).

In the recent years, targeting the so-called Immune Checkpoint molecules (ICs) which provide inhibitory signals to $\mathrm{T}$ cells, has offered new exciting treatment opportunities in cancer (8). Inhibition of autoreactive $\mathrm{CD}^{+}$T-cells through ICs is a physiological mechanism to prevent autoimmunity; on the other end, this mechanism inhibits the immune response against aberrant cancer cells. Differently from conventional cytotoxic or from targeted therapies that are aimed at the cancer cells, the therapies that involve the modulation of ICs attempt to redirect the function of the immune system to elicit cancer cell death. Several checkpoint molecules capable to shut down the response against neo-antigens are present on $\mathrm{T}$ cells as well as on tumor cells. These molecules are at the center of regulatory networks that result in immunosuppression. Antibodies against the "classic" IC molecules (CTLA-4, PD-1, PD-L1, and PD-L2) are considered the "first generation" IC inhibitors (ICis) that interfere with the immune escape of tumor cells, followed by second and third generations ICis targeting other immunoregulatory molecules and pathways $(9,10)$.

Immune checkpoints inhibition dramatically improved survival in hard-to-treat tumors like lung cancer and melanoma so that the therapy with IC inhibitors (ICis) has entered in the standard clinical practice for these tumors whereas clinical trials have been launched for many other tumors $(8,10)$.

\section{BLOOD-BRAIN BARRIER, IMMUNOLOGICAL MECHANISMS AND IMMUNE CHECKPOINTS INTERPLAY IN GLIOBLASTOMA}

For many years the CNS has been considered as an immuneprivileged compartment with the $\mathrm{BBB}$ responsible to maintain a constant brain microenvironment from metabolic insults and, at the same time, physically blocking or actively favoring the transport of bioactive molecules. During the development of glioma, the integrity of the $\mathrm{BBB}$ is preserved up to a tumor size of $\sim 2 \mathrm{~mm}^{3}$; above that, the angiogenetic pressure elicited by GBM releases the tight and adherent junctions between the cerebral endothelial cells allowing the passage of molecules up to $12 \mathrm{~nm}(11,12)$. With further tumor growth the BBB becomes freely permeable to larger molecules. Nevertheless, tumor cells in niches at the boundary of the surgically excised tumor remain protected by the $\mathrm{BBB}$ reducing the efficacy of the treatment. Beside immune cells (13), several cell types in the brain (microglia, astrocytes) can act as antigen-presenting cells and elicit immune response against the tumor. This mechanism is aided by the permeability of the damaged $\mathrm{BBB}$ that enables the passage of tumor antigens outside the brain (14-16). Similarly to other tumors, GBM is associated with significant immunosuppression particularly in the T-cell compartment (17) because of the combined effect of steroid/cytotoxic treatment, the downregulation of MHC-I antigens and of the secretion of immunosuppressive cytokines. Moreover, also in glioma, the correct maintenance of the physiological status of immunological tolerance and response is mediated by the coordinate interplay of many actors, including IC molecules, and different cell types as summarized below and in Figure 1.

\section{CTLA-4}

CTLA-4 (CD152) was the first immunoregulatory molecule to be targeted for therapeutic purposes utilizing the humanized antibody Ipilimumab approved by FDA and EMA in 2011, initially for melanoma (18), soon followed by Tremelimumab for mesothelioma $(19,20)$. CTLA-4, expressed on T-cells (activated and regulatory), interacts with its ligands CD80 and CD86 on APCs to inhibit co-stimulators T-cell pathways (21). In GB the expression of CTLA-4 on $\mathrm{CD}^{+}$and $\mathrm{CD}^{+}$cells is strongly inversely correlated with outcome (22).

\section{PD-1/PD-L1}

PD-1 on T cells and its ligand PD-L1 on APC and tumor cells are the most important immunosuppressive molecules so far identified. Their interaction leads to the suppression of early Tcell activation, abolishing their cytotoxic activity and interferes with the production of inflammatory cytokines $(23,24)$. Two PD-1 suppressive Ab were licensed in 2014 (Nivolumab) and in 2016 (Pembrolizumab) and two anti-PD-L1 Ab: Atezolizumab in 2016 and Avelumab in 2017. Their original indications were rapidly extended to other tumors and many clinical trials with newer molecules are ongoing $(8,25,26)$. The expression of PD-L1 on glioma cells has been documented as well as that of PD-1 on tumor infiltrating lymphocytes (TIL). The functional and clinical implications of PD-1/PD-L1 expression in GBM are still unclear. Indeed, no correlation between PD-L1 expression and overall survival was seen in two cohorts $(27,28)$. On the other hand, in another study, PD-L1 staining and PD1/PD-L1 expression were associated with decreased survival (29). The absence of standardized experimental parameters, of defined cut-off values and the heterogeneity of the cohorts might explain these contrasting findings. Interestingly PD-L1 expression is directly correlated with WHO grade and within Grade IV tumors, PD-L1 expression is significantly higher in IDH1/2 wt tumors compared to IDH1/2 mutated or hyper methylated GBM (27). Overall, the expression of PD-L1 is linked to well-known negative prognostic indicators in GBM and its effect on survival must be examined in homogeneous cohorts.

\section{Tim-3}

Tim 3 is a molecule expressed by CD4+ and CD8+ T cells that, similarly to PD-1, is involved in immune suppression and promotes tumor escape through the exhaustion of T cells (30). A large proportion of TILs in GBM and other tumors is composed by $\mathrm{T}$ cells not capable of cytokine secretion and not exerting their physiological function. In GBM the overexpression of TIM3 is associated with higher malignancy (higher grade, lower 


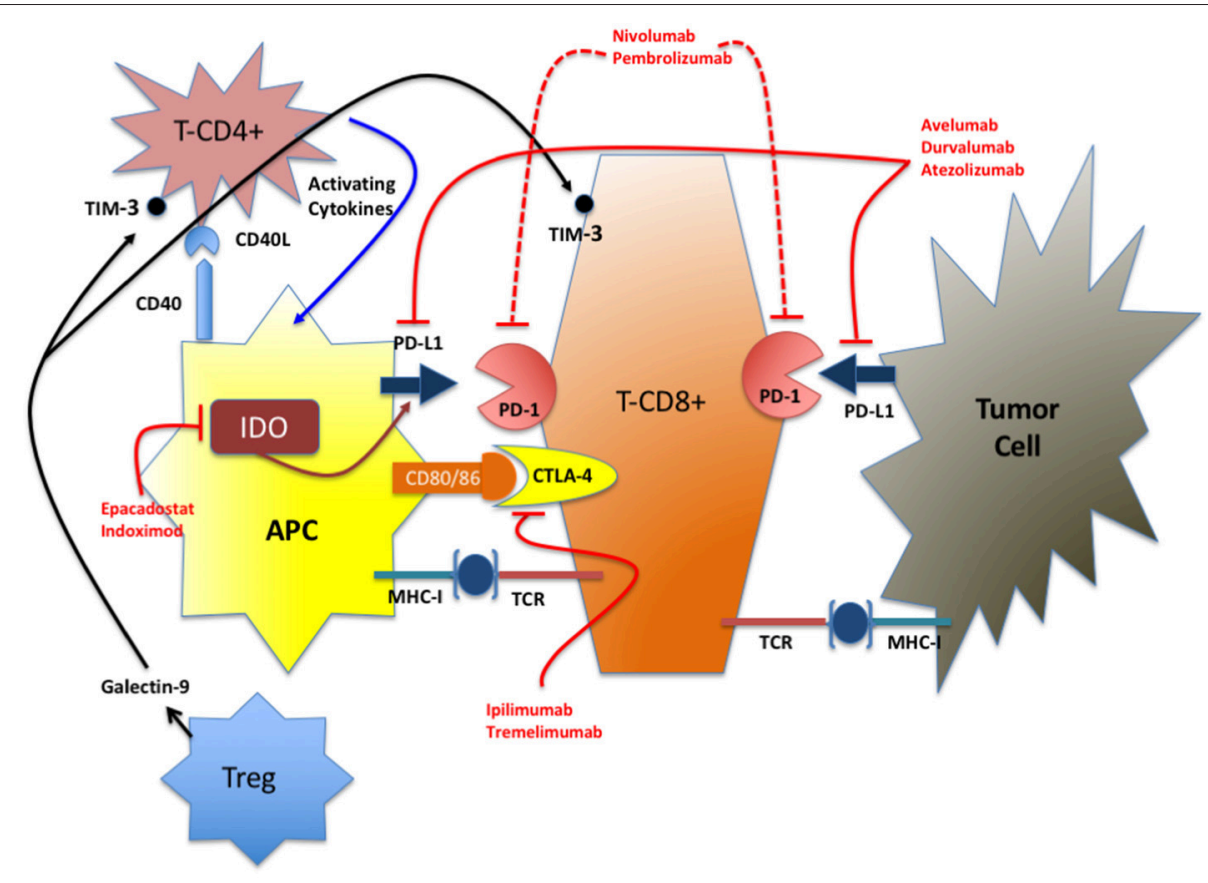

FIGURE 1 | Simplified representation of the IC network. In red are indicated the FDA-approved drugs and the IDO inhibitors in advanced stage of clinical test (phase III). TIM-3 inhibitors are at an early stage of development for clinical use (phase I).

Karnofsky score, and IDHwt) and is thus considered a strong negative prognostic indicator $(31,32)$.

\section{IDO}

Although IDO is not a classical immune checkpoint molecule and lacks receptorial capacity, it is included in this functional class because of its suppressive properties on T-cell activation and NK cell function (33). Similarly to TIM-3, IDO overexpression is linked to poorer outcome in GBM patients (34) and targeting IDO with Epacadostat or Indoximod (35), was a successful experimental strategy in in vivo models (36).

\section{GENETIC AND EPIGENETIC FACTORS DETERMINE THE FUNCTIONALITY OF IC MOLECULES}

Targeting IC molecules with blocking antibodies alone or in combination with other ICi therapies, or with standard chemotherapy has revolutionized the therapeutic approach to lung cancer and other hard to treat tumors. Nevertheless, along with very favorable response rate, other patients are unresponsive to the therapy or show life-threatening side effects. Predicting the response and the appearance of major side effects during treatment is a major health issue. Limiting certain therapies to patients likely to respond and strict monitoring of patients at risk have several ethical implications and could enable the Public Health Systems to offer the best available therapy to the patients who could really benefit from it. However, reliable biomarkers predicting response or adverse reactions to ICi therapy are not yet available.

Single nucleotide variations (SNVs) of IC genes can affect the expression levels of IC molecules thus altering immune tolerance and leading to increased susceptibility to autoimmune diseases or to reduced immunological response against cancer cells.

A meta-analysis that included 12 studies and more than 5,000 tumor patients and an equal number of healthy controls showed a decreased cancer risk for TT homozygous individuals at polymorphism PD-1.5 (rs2227981) and, for Asian populations, a decreased risk was seen for AG individuals and an increased risk for AA, at PD-1.3 (rs11568821) (37). PD-1.5 allele frequencies and risk of low- and high-grade glioma development were examined in 156 mid-Eastern patients and significantly higher frequency of the PD1.5 C/T and T/T genotype were found in high-grade glioma compared to low-grade tumors and control individuals (38).

Some studies, described below, have examined the clinical impact of IC polymorphisms in tumor and autoimmune disease patients treated with ICi. Years ago, it was shown that SNVs in CTLA-4 may affect the transcriptional activity of the gene as well as the interaction with CD80 and influence immune response in autoimmune diseases $(39,40)$. SNV of CTLA-4 are implicated in clinical response and survival in melanoma patients $(41,42)$ and, more recently, SNV-1577G > A and SNV CT60G > A were linked to a better response to Ipilimumab in a 173-patients cohort (43) and SNV-1661A > G to the onset of endocrine adverse events (44). 
In NSCLC CTLA-4, $P D-1$, and $P D-L 1$ were examined in two studies that involved more than 400 patients $(21,45)$. Overall three PD-L1 SNV (rs2282055G > T; rs4143815C > G, and rs2297136T > C) were significantly associated with a better overall response rate and improved OS and PFS when treated with chemotherapy alone or with Nivolumab as second or third line of treatment after chemotherapy.

Overall these results indicate that genetic variations in IC molecules can be utilized as conventional biomarkers predictive of response to treatment and outcome to optimize patients' treatment. This is particularly important also in view of the availability of new drugs whose efficacy and toxicity may be genetically-dependent and whose utilization requires a "personalized approach" to cancer treatment.

Studies on hematologic disorders like myelodysplastic syndrome (MDS) treated with inhibitors of DNA methyltransferases demonstrated the up-regulation of PD-1 as a consequence of the therapy $(46,47)$. High expression of $\mathrm{PD}-1$ is a negative prognostic indicator and it has been proposed that the treatment of MDS with hypomethylating agents should be coupled with the blockade of ICs.

The analysis of several solid tumors demonstrated that epigenetic mechanisms regulate the expression of IC molecules and that methylation of PD-1 and PD-L1 promoters is associated with worse outcome (48-51).

The immunological landscape of glioma is influenced by IDH1/2 mutations; indeed, in mutated tumors PD-L1 is significantly diminished supporting the rationale of ICi treatment in IDH1/2wt patients (52). Besides DNA methylation, other epigenetic modifiers may influence IC expression in glioma. Namely, miRNAs may directly impact CTLA-4, PD-1, and PDL1 expression through a series of miRNA like mir-155 (CTLA4), mir-138 (CTLA-4 and PD-1), miR-424 (PD-L1 and CD80), mir-28 (PD-1), miR-34a, miR-200 miR-513, and miR-138-5p (PD-L1).

Moreover, the same or other miRNA regulate the expression of cytokines like IFN- $\gamma$ or transcription factors that are positive or negative regulators of IC generating a redundant and extremely complex network. The interaction between IC molecules, and miRNA have been recently shortly reviewed (53).

\section{IMMUNE CHECKPOINT BLOCKADE IN GBM: PRECLINICAL FINDINGS}

Several preclinical trials conducted utilizing two immunocompetent animal models (GL261/C57Bl/6 and SMA560/VM/dk) (54) demonstrated that IC blockade utilizing ICi as single agent or in combination significantly prolongs survival at an extent that depends on the molecule, or their combination. In one study, CTLA- 4 blockade alone resulted in $80 \%$ of long survivors (55) whereas in two others the percentage of long survivors was 40 and $25 \%(28,56)$. PD-1 blockade alone resulted in 56\% long survivors in one study (57) but had no effect in another study unless associated with radiotherapy (15-40\% long survivors) (58). PD-L1 blockade was tested in two studies leading to $60 \%$ (57) and 25\% long survivors (56). Only one study examined the effect of TIM-3 blockade with no effect on survival (59). The effect of the therapy was strongly augmented when different ICis were utilized in combination or with standard therapy. Two studies in murine models demonstrated that the combination of radiation therapy and $\mathrm{PD}-1$ and/or TIM-3 exerted a strong antitumor response over the treatment with a single agent and the maximal activity (100\% long survivors) was seen when PD-1 and TIM-3 inhibition were combined with stereotactic radiosurgery $(58,59)$. Another study described the effects of the concomitant CTLA-4/PD-L1/PD-L2 inhibition that resulted in $75 \%$ long survival (56). Finally, disabling the entire IC network (CTLA-4/PD-L1/IDO) (57) or the dual IC blockade (TIM-3/PD-1) coupled with radiosurgery (59), both resulted in the survival of $100 \%$ of the treated mice. Importantly in all these treatments it was possible to demonstrate the activation of the immune system within the tumor (cytokines production, TIL activation, etc.) and sustained anti-tumor immune response since regrowth of the tumor was not observed after tumor cell re-challenge.

Overall, these preclinical models support the rationale for disabling many components of the IC network in conjunction with standard therapies for an efficient glioma treatment. Moreover, since the concomitant utilization of several ICis could increase the risk of life threatening adverse effects, the need of identifying molecular markers predicting response and therapy-induced toxicity, as previously mentioned, appears of the utmost importance for the clinical utilization of combined IC treatment.

\section{IMMUNE CHECKPOINT BLOCKADE IN GBM: CLINICAL TRIALS}

The successful preclinical trials and the very favorable results obtained with other tumors like NSCLC and melanoma, set the basis for the utilization of ICis in many other tumors including GBM. The survey of the NIH Clinical Trials Database (https://www.clinicaltrials.gov) performed on July 2018, showed 60 registered trials; only two of them were completed (Table 1 ). One of the completed studies (NCT01860638) is a phase II randomized study to test the safety of the combination Bevacizumab/Lomustine as second line treatment followed by Nivolumab as third line. The primary endpoint of the study that enrolled 296 patients and ended in 2017 was OS but the results were not made available to the public. The second completed study was NCT02550249, a phase II study that enrolled 29 patients and had as primary outcome the evaluation of the expression of PD-L1 in tumor cells and lymphocytes upon treatment with Nivolumab. Also, in this case the results are not available.

The ongoing studies (mostly phase I or II) test several ICi molecules as single agents or in various combinations with standard cytotoxic molecules, targeted therapies, or other immunological therapies and are aimed, not only at determining the clinical utility of these molecules, but also 
TABLE 1 | Clinical trials with IC inhibitors in glioma (July, 2018).

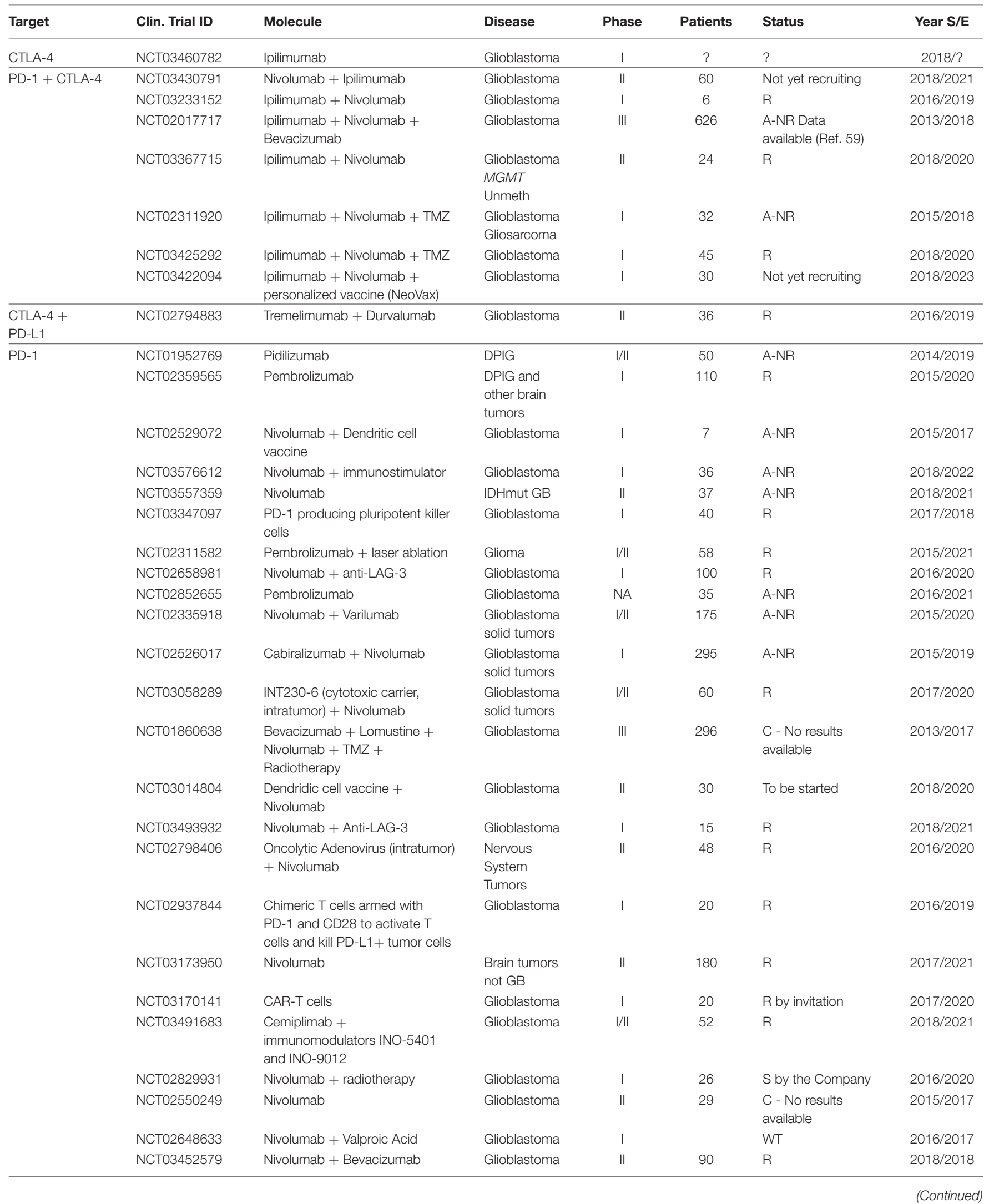


TABLE 1 | Continued

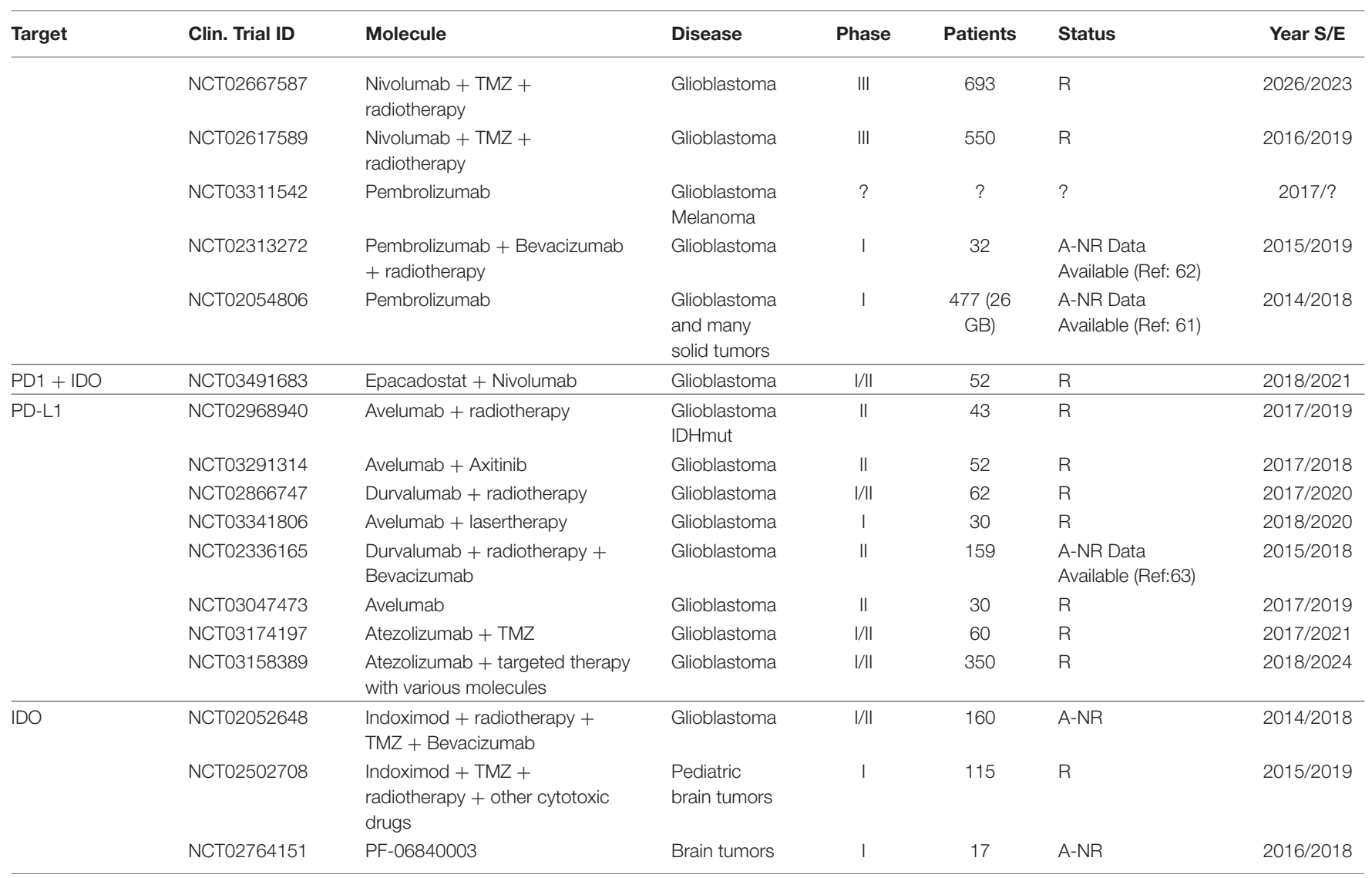

Data taken from https://www. clinicaltrials.gov

R, Recruiting; C, Completed; A-NR, Active Not Recruiting; AC, Accrual completed; NI, Not Indicated; WT, Withdrawn; S, Suspended; Year S/E, Year Start/End.

at determining the safety of the treatment and are expected to be completed starting from 2019 but mostly after year 2020.

The only study with published results is NCT02017717 (CheckMate 143), a large phase III randomized trial that enrolled over 600 patients. This study was the first large trial where the effect of IC inhibitors was stringently tested. Encouraging results were initially obtained in one of the study arms where three patients showed partial response and 8 disease stabilization with the combination Nivolumab+Ipilimumab (60), however when the study was extended, this arm was closed because of the treatment failure (61).

Two other large phase III randomized trials (NCT02617589 and NCT02667587) are testing the effect of Nivolumab on MGMT methylated or unmethylated patients and the results of these studies are expected in 2019 and 2023, respectively.

Phase Ib trial NCT02054806 tested the safety and efficacy of the PD-1 inhibitor Pembrolizumab on a large series of solid tumors. In the GBM arm (26 patients), one partial response and 12 disease stabilization were observed (62).

Phase I trial NCT02313272 tested the effect of the addition of Pembrolizumab to Bevacizumab and radiotherapy. The initial results were encouraging since more than $50 \%$ of the patients at 6 months showed partial or complete response (63).

Finally, phase 2 trial NCT02336165, the PD-1 inhibitor Durvalumab was tested in combination with Bevacizumab and radiotherapy and showed partial response or disease stabilization in $60 \%$ of the patients after 6 months. Four patients remained progression free (64).

\section{CONCLUSIONS}

Targeting ICs has revolutionized the therapeutic approach to certain tumors. There is strong hope that this therapy could be effective also for GBM patients. Indeed, the preclinical trials and the initial results obtained in some phase I/II studies suggested that ICis could offer new therapeutic options to these patients. The results of the first large phase III trials were somehow disappointing and inhibiting PD-1 could not fully restore the host immune response (61).

Nevertheless, the treatment with Nivolumab doubled the response to therapy in $8 \%$ of the patients (11.1 months vs. 5.3 with Bevacizumab) (61). Moreover, the high levels of VEGF seen in GBM are strongly immunosuppressive and this effect should be better counteracted. In this respect, targeting multiple IC pathways also in combination with cytotoxic drugs could be 
a winning strategy. The results of the two ongoing phase III trials and of the phase I/II trials where combination therapies are explored may provide new weapons against this rapidly and invariably deadly cancer.

\section{AUTHOR CONTRIBUTIONS}

MR conceived the idea of this mini-review article and wrote the first draft. BB, MP, RC, AM, and MR equally participated to the final writing of the article.

\section{REFERENCES}

1. Stupp R, Mason WP, van den Bent MJ, Weller M, Fisher B, Taphoorn MJB, et al. Radiotherapy plus concomitant and adjuvant temozolomide for glioblastoma. N Engl J Med. (2005) 352:987-96. doi: 10.1056/NEJMoa043330

2. Puduvalli VK, Chaudhary R, McClugage SG, Markert J. Beyond alkylating agents for gliomas: quo vadimus? Am Soc Clin Oncol Educ Book (2017) 37:175-86. doi: 10.14694/EDBK_175003

3. Staedtke V, Bai R-Y, Laterra J. Investigational new drugs for brain cancer. Expert Opin Invest Drugs (2016) 25:937-56. doi: 10.1080/13543784.2016.1182497

4. Chen R, Cohen AL, Colman H. Targeted therapeutics in patients with highgrade gliomas: past, present, and future. Curr Treat Options Oncol. (2016) 17:42. doi: 10.1007/s11864-016-0418-0

5. Touat M, Idbaih A, Sanson M, Ligon KL. Glioblastoma targeted therapy: updated approaches from recent biological insights. Ann Oncol. (2017) 28:1457-72. doi: 10.1093/annonc/mdx106

6. Ellis HP, Greenslade M, Powell B, Spiteri I, Sottoriva A, Kurian KM. Current challenges in glioblastoma: intratumour heterogeneity, residual disease, and models to predict disease recurrence. Front Oncol. (2015) 5:251. doi: $10.3389 /$ fonc. 2015.00251

7. Ramirez Y, Weatherbee J, Wheelhouse R, Ross A. Glioblastoma multiforme therapy and mechanisms of resistance. Pharmaceuticals (2013) 6:1475-506. doi: 10.3390/ph6121475

8. Bersanelli M, Buti S. From targeting the tumor to targeting the immune system: transversal challenges in oncology with the inhibition of the PD-1/PDL1 axis. World J Clin Oncol. (2017) 8:37. doi: 10.5306/wjco.v8.i1.37

9. Callahan MK, Postow MA, Wolchok JD. CTLA-4 and PD-1 pathway blockade: combinations in the clinic. Front Oncol. (2015) 4:385. doi: 10.3389/fonc.2014.00385

10. Dempke WCM, Fenchel K, Uciechowski P, Dale SP. Second- and thirdgeneration drugs for immuno-oncology treatment-the more the better? Eur J Cancer (2017) 74:55-72. doi: 10.1016/j.ejca.2017.01.001

11. Davies DC. Blood-brain barrier breakdown in septic encephalopathy and brain tumours*. J Anatomy (2002) 200:639-46. doi: 10.1046/j.1469-7580.2002.00065.x

12. Schlageter KE, Molnar P, Lapin GD, Groothuis DR. Microvessel organization and structure in experimental brain tumors: microvessel populations with distinctive structural and functional properties. Microvasc Res. (1999) 58:31228. doi: 10.1006/mvre.1999.2188

13. Prins RM, Soto H, Konkankit V, Odesa SK, Eskin A, Yong WH, et al. Gene expression profile correlates with T-cell infiltration and relative survival in glioblastoma patients vaccinated with dendritic cell immunotherapy. Clin Cancer Res. (2011) 17:1603-15. doi: 10.1158/1078-0432.CCR-10-2563

14. Jarry U, Jeannin P, Pineau L, Donnou S, Delneste Y, Couez D. Efficiently stimulated adult microglia cross-prime naive $\mathrm{CD} 8+\mathrm{T}$ cells injected in the brain: antigen processing. Eur J Immunol. (2013) 43:1173-84. doi: 10.1002/eji.201243040

15. Aloisi F, Ria F, Adorini L. Regulation of T-cell responses by CNS antigenpresenting cells: different roles for microglia and astrocytes. Immunol Today (2000) 21:141-7. doi: 10.1016/S0167-5699(99)01512-1

16. Becher B, Bechmann I, Greter M. Antigen presentation in autoimmunity and CNS inflammation: how T lymphocytes recognize the brain. J Mol Med. (2006) 84:532-43. doi: 10.1007/s00109-006-0065-1

\section{FUNDING}

This study was supported by the Italian Ministry of Health, Core Grant Ricerca Corrente to the IRCCS Ospedale Policlinico San Martino, Genova, by the grant from the Fondazione Compagnia di San Paolo-Torino: Terapie innovative per il glioblastoma and by the grant Associazione Italiana Ricerca sul Cancro (AIRC) $5 \times 1000$ N. 21073 Epigenetic modeling/remodeling of cancer metastases and tumor immune contexture to improve efficacy of immunotherapy.

17. Fecci PE, Mitchell DA, Whitesides JF, Xie W, Friedman AH, Archer GE, et al Increased regulatory $\mathrm{T}$-cell fraction amidst a diminished CD4 compartment explains cellular immune defects in patients with malignant glioma. Cancer Res. (2006) 66:3294-302. doi: 10.1158/0008-5472.CAN-05-3773

18. Hodi FS, O'Day SJ, McDermott DF, Weber RW, Sosman JA, Haanen JB, et al. Improved survival with ipilimumab in patients with metastatic melanoma. $N$ Engl J Med. (2010) 363:711-23. doi: 10.1056/NEJMoa1003466

19. Ibarrondo FJ, Comin-Anduix B, Escuin-Ordinas H. Tremelimumab: research and clinical development. OncoTargets Ther. (2016) 2016:1767. doi: 10.2147/OTT.S65802

20. Calabrò L, Morra A, Fonsatti E, Cutaia O, Fazio C, Annesi D, et al. Efficacy and safety of an intensified schedule of tremelimumab for chemotherapyresistant malignant mesothelioma: an open-label, single-arm, phase 2 study. Lancet Respir Med. (2015) 3:301-9. doi: 10.1016/S2213-2600(15)00092-2

21. Lee K-M, Chuang E, Griffin M, Khattri R, Hong DK, Zhang W, et al. Molecular basis of T cell inactivation by CTLA-4. Science (1998) 282:2263-6. doi: 10.1126/science.282.5397.2263

22. Fong B, Jin R, Wang X, Safaee M, Lisiero DN, Yang I, et al. Monitoring of regulatory $\mathrm{T}$ cell frequencies and expression of CTLA- 4 on T cells, before and after DC vaccination, can predict survival in GBM patients. PLoS ONE (2012) 7:e32614. doi: 10.1371/journal.pone.0032614

23. Cheng X, Veverka V, Radhakrishnan A, Waters LC, Muskett FW, Morgan $\mathrm{SH}$, et al. Structure and interactions of the human programmed cell death 1 receptor. J Biol Chem. (2013) 288:11771-85. doi: 10.1074/jbc.M112. 448126

24. Francisco LM, Salinas VH, Brown KE, Vanguri VK, Freeman GJ, Kuchroo VK, et al. PD-L1 regulates the development, maintenance, and function of induced regulatory T cells. J Exp Med. (2009) 206:3015-29. doi: 10.1084/jem.20090847

25. Preusser M, Lim M, Hafler DA, Reardon DA, Sampson JH. Prospects of immune checkpoint modulators in the treatment of glioblastoma. Nat Rev Neurol. (2015) 11:504-14. doi: 10.1038/nrneurol.2015.139

26. Mahmoudi M, Farokhzad OC. Cancer immunotherapy: woundbound checkpoint blockade. Nat Biomed Eng. (2017) 1:0031. doi: 10.1038/s41551-017-0031

27. Heiland DH, Haaker G, Delev D, Mercas B, Masalha W, Heynckes S, et al. Comprehensive analysis of PD-L1 expression in glioblastoma multiforme. Oncotarget (2017) 8:15031. doi: 10.18632/oncotarget.15031

28. Wang Z, Zhang C, Liu X, Wang Z, Sun L, Li G, et al. Molecular and clinical characterization of PD-L1 expression at transcriptional level via 976 samples of brain glioma. OncoImmunology (2016) 5:e1196310. doi: 10.1080/2162402X.2016.1196310

29. Nduom EK, Wei J, Yaghi NK, Huang N, Kong L-Y, Gabrusiewicz K, et al. PDL1 expression and prognostic impact in glioblastoma. Neurooncology (2016) 18:195-205. doi: 10.1093/neuonc/nov172

30. Das M, Zhu C, Kuchroo VK. Tim-3 and its role in regulating anti-tumor immunity. Immunol Rev. (2017) 276:97-111. doi: 10.1111/imr.12520

31. Han S, Feng S, Xu L, Shi W, Wang X, Wang H, et al. Tim-3 on peripheral CD4+ and CD8+ T cells is involved in the development of glioma. DNA Cell Biol. (2014) 33:245-50. doi: 10.1089/dna.2013.2306

32. Li G, Wang Z, Zhang C, Liu X, Cai J, Wang Z, et al. Molecular and clinical characterization of TIM-3 in glioma through 1,024 samples. OncoImmunology (2017) 6:e1328339. doi: 10.1080/2162402X.2017.1328339

33. Prendergast GC, Smith C, Thomas S, Mandik-Nayak L, Laury-Kleintop L, Metz R, et al. Indoleamine 2,3-dioxygenase pathways of pathogenic 
inflammation and immune escape in cancer. Cancer Immunol Immunother. (2014) 63:721-35. doi: 10.1007/s00262-014-1549-4

34. Sordillo PP, Sordillo LA, Helson L. The kynurenine pathway: a primary resistance mechanism in patients with glioblastoma. Anticancer Res. (2017) 37:2159-71. doi: 10.21873/anticanres.11551

35. Cheong JE, Ekkati A, Sun L. A patent review of IDO1 inhibitors for cancer. Expert Opin Ther Pat. (2018) 28:317-30. doi: 10.1080/13543776.2018.1441290

36. Hanihara M, Kawataki T, Oh-Oka K, Mitsuka K, Nakao A, Kinouchi H. Synergistic antitumor effect with indoleamine 2,3-dioxygenase inhibition and temozolomide in a murine glioma model. J Neurosurg. (2015) 124:1594-601. doi: 10.3171/2015.5.JNS141901

37. Dong W, Gong M, Shi Z, Xiao J, Zhang J, Peng J. Programmed cell death-1 polymorphisms decrease the cancer risk: a meta-analysis involving twelve case-control studies. PLOS ONE (2016) 11:e0152448. doi: 10.1371 /journal.pone. 0152448

38. Jahromi FN, Samadi M, Mojtahedi Z, Haghshenas MR, Taghipour M, Erfani N. Association of PD-1.5 C/T, but Not PD-1.3 G/A, with malignant and benign brain tumors in iranian patients. Immunol Invest. (2017) 46:469-80. doi: 10.1080/08820139.2017.1296858

39. Anjos S, Nguyen A, Ounissi-Benkalha H, Tessier M-C, Polychronakos C. A common autoimmunity predisposing signal peptide variant of the cytotoxic T-lymphocyte antigen 4 results in inefficient glycosylation of the susceptibility allele. J Biol Chem. (2002) 277:46478-86. doi: 10.1074/jbc.M206894200

40. Ueda H, Howson JMM, Esposito L, Heward J, Snook, Chamberlain G, et al. Association of the T-cell regulatory gene CTLA4 with susceptibility to autoimmune disease. Nature (2003) 423:506-11. doi: 10.1038/nature01621

41. Breunis WB, Tarazona-Santos E, Chen R, Kiley M, Rosenberg SA, Chanock SJ. Influence of cytotoxic $\mathrm{T}$ lymphocyte-associated antigen 4 (CTLA4) common polymorphisms on outcome in treatment of melanoma patients with CTLA-4 blockade. J Immunother. (2008) 31:586-90. doi: 10.1097/CJI.0b013e31817fd8f3

42. Queirolo P, Morabito A, Laurent S, Lastraioli S, Piccioli P, Ascierto PA, et al. Association of CTLA-4 polymorphisms with improved overall survival in melanoma patients treated with CTLA-4 blockade: a pilot study. Cancer Invest. (2013) 31:336-45. doi: 10.3109/07357907.2013.793699

43. Queirolo P, Dozin B, Morabito A, Banelli B, Piccioli P, Fava C, et al. Association of CTLA-4 gene variants with response to therapy and longterm survival in metastatic melanoma patients treated with ipilimumab: an italian melanoma intergroup study. Front Immunol. (2017) 8:386. doi: 10.3389/fimmu.2017.00386

44. Queirolo P, Dozin B, Morabito A, Banelli B, Carosio R, Fontana V, et al. CTLA4 gene variant $-1661 \mathrm{~A}>\mathrm{G}$ may predict the onset of endocrine adverse events in metastatic melanoma patients treated with ipilimumab. Eur J Cancer (2018) 97:59-61. doi: 10.1016/j.ejca.2018.04.005

45. Nomizo T, Ozasa H, Tsuji T, Funazo T, Yasuda Y, Yoshida $H$, et al. Clinical impact of single nucleotide polymorphism in PD-L1 on response to nivolumab for advanced non-small-cell lung cancer patients. Sci Rep. (2017) 7:45124. doi: 10.1038/srep45124

46. Yang H, Bueso-Ramos C, DiNardo C, Estecio MR, Davanlou M, Geng QR, et al. Expression of PD-L1, PD-L2, PD-1 and CTLA4 in myelodysplastic syndromes is enhanced by treatment with hypomethylating agents. Leukemia (2014) 28:1280-8. doi: 10.1038/leu.2013.355

47. Polivka J Jr, Polivka J, Holubec L, Kubikova T, Priban V, Hes O, et al. Advances in experimental targeted therapy and immunotherapy for patients with glioblastoma multiforme. Anticancer Res. (2017) 37:11285. doi: 10.21873/anticanres. 11285

48. Gevensleben H, Holmes EE, Goltz D, Dietrich J, Sailer V, Ellinger J, et al. PD-L1 promoter methylation is a prognostic biomarker for biochemical recurrencefree survival in prostate cancer patients following radical prostatectomy. Oncotarget (2016) 7:13161. doi: 10.18632/oncotarget.13161

49. Sakaki M, Ebihara Y, Okamura K, Nakabayashi K, Igarashi A, Matsumoto K, et al. Potential roles of DNA methylation in the initiation and establishment of replicative senescence revealed by array-based methylome and transcriptome analyses. PLOS ONE (2017) 12:e0171431. doi: 10.1371/journal.pone.0171431

50. Bally APR, Austin JW, Boss JM. Genetic and epigenetic regulation of PD-1 expression. J Immunol. (2016) 196:2431-7. doi: 10.4049/jimmunol. 1502643
51. Goltz D, Gevensleben H, Dietrich J, Dietrich D. PD-L1 (CD274) promoter methylation predicts survival in colorectal cancer patients. OncoImmunology (2017) 6:e1257454. doi: 10.1080/2162402X.2016.1257454

52. Mu L, Long Y, Yang C, Jin L, Tao H, Ge H, et al. The IDH1 mutationinduced oncometabolite, 2-hydroxyglutarate, may affect DNA methylation and expression of PD-L1 in gliomas. Front Mol Neurosci. (2018) 11:82. doi: $10.3389 /$ fnmol.2018.00082

53. Banelli B, Forlani A, Allemanni G, Morabito A, Pistillo MP, Romani M. MicroRNA in glioblastoma: an overview. Int J Genomics (2017) 2017:1-16. doi: $10.1155 / 2017 / 7639084$

54. Oh T, Fakurnejad S, Sayegh ET, Clark AJ, Ivan ME, Sun MZ, et al. Immunocompetent murine models for the study of glioblastoma immunotherapy. J Trans Med. (2014) 12:107. doi: 10.1186/1479-5876-12-107

55. Fecci PE, Ochiai H, Mitchell DA, Grossi PM, Sweeney AE, Archer GE, et al. Systemic CTLA-4 blockade ameliorates glioma-induced changes to the CD4+ $\mathrm{T}$ cell compartment without affecting regulatory T-cell function. Clin Cancer Res. (2007) 13:2158-67. doi: 10.1158/1078-0432.CCR-06-2070

56. Reardon DA, Gokhale PC, Klein SR, Ligon KL, Rodig SJ, Ramkissoon SH, et al. Glioblastoma eradication following immune checkpoint blockade in an orthotopic, immunocompetent model. Cancer Immunol Res. (2016) 4:124-35. doi: 10.1158/2326-6066.CIR-15-0151

57. Wainwright DA, Chang AL, Dey M, Balyasnikova IV, Kim CK, Tobias A, et al. Durable therapeutic efficacy utilizing combinatorial blockade against IDO, CTLA-4, and PD-L1 in mice with brain tumors. Clin Cancer Res. (2014) 20:5290-301. doi: 10.1158/1078-0432.CCR-14-0514

58. Zeng J, See AP, Phallen J, Jackson CM, Belcaid Z, Ruzevick J, et al. AntiPD-1 blockade and stereotactic radiation produce long-term survival in mice with intracranial gliomas. Int J Radiat Oncol Biol Phys. (2013) 86:343-9. doi: 10.1016/j.ijrobp.2012.12.025

59. Kim JE, Patel MA, Mangraviti A, Kim ES, Theodros D, Velarde E, et al. Combination therapy with anti-PD-1, anti-TIM-3, and focal radiation results in regression of murine gliomas. Clin Cancer Res. (2017) 23:124-36. doi: 10.1158/1078-0432.CCR-15-1535

60. Reardon DA, Sampson JH, Sahebjam S, Lim M, Baehring JM, Vlahovic G, et al. Safety and activity of nivolumab (nivo) monotherapy and nivo in combination with ipilimumab (ipi) in recurrent glioblastoma (GBM): updated results from checkmate-143. J. Clin Oncol. (2016) 34:2014. doi: 10.1200/JCO.2016.34.15_suppl.2014

61. Filley AC, Henriquez M, Dey M. Recurrent glioma clinical trial, CheckMate-143: the game is not over yet. Oncotarget (2017) 8:21586. doi: $10.18632 /$ oncotarget. 21586

62. Reardon DA, Kim T-M, Frenel J-S, Santoro A, Lopez J, Subramaniam DS, et al. ATIM-35. Results of the phase ib keynote-028 multi-cohort trial of pembrolizumab monotherapy in patients with recurrent pd-11positive glioblastoma multiforme (GBM). Neuro Oncol. (2016) 18:vi25-6. doi: 10.1093/neuonc/now212.100

63. Sahebjam S, Forsyth P, Arrington J, Jaglal M, Tran ND, Etame AB, et al. ATIM-18. A phase $\mathrm{i}$ trial of hypofractionated stereotactic irradiation (hfsrt) with pembrolizumab and bevacizumab in patients with recurrent high grade glioma (NCT02313272). Neuro Oncol. (2017) 19:vi30. doi: $10.1093 /$ neuonc/nox 168.113

64. Reardon DA, Kaley TJ, Dietrich J, Clarke JL, Dunn GP, Lim M, et al. Phase 2 study to evaluate safety and efficacy of MEDI4736 (durvalumab [DUR]) in glioblastoma (GBM) patients: an update. J. Clin Oncol. (2017) 35:2042. doi: 10.1200/JCO.2017.35.15_suppl.2042

Conflict of Interest Statement: The authors declare that the research was conducted in the absence of any commercial or financial relationships that could be construed as a potential conflict of interest.

Copyright $\odot 2018$ Romani, Pistillo, Carosio, Morabito and Banelli. This is an openaccess article distributed under the terms of the Creative Commons Attribution License (CC BY). The use, distribution or reproduction in other forums is permitted, provided the original author(s) and the copyright owner(s) are credited and that the original publication in this journal is cited, in accordance with accepted academic practice. No use, distribution or reproduction is permitted which does not comply with these terms. 